\title{
Provider Experiences with Chronic Care Management (CCM) Services and Fees: A Qualitative Research Study
}

\author{
Ann S. O'Malley, $M D, M P H^{7}$, Rumin Sarwar, $\mathrm{MS}^{7}$, Rosalind Keith, $P D^{7}$, Patrick Balke, BA', \\ Sai Ma, $P h D^{2}$, and Nancy McCall, $S c D^{7}$ \\ ${ }^{7}$ Mathematica Policy Research, Washington, DC, USA; ${ }^{2}$ The Innovation Center (CMMI), Centers for Medicare \& Medicaid Services (CMS), \\ Baltimore, MD, USA.
}

BACKGROUND: Support for ongoing care management and coordination between office visits for patients with multiple chronic conditions has been inadequate. In January 2015, Medicare introduced the Chronic Care Management (CCM) payment policy, which reimburses providers for CCM activities for Medicare beneficiaries occurring outside of office visits.

OBJECTIVE: To explore the experiences, facilitators, and challenges of practices providing CCM services, and their implications going forward.

DESIGN: Semi-structured telephone interviews from January to April 2016 with 71 respondents.

PARTICIPANTS: Sixty billing and non-billing providers and practice staff knowledgeable about their practices' CCM services, and 11 professional society representatives. KEY RESULTS: Practice respondents noted that most patients expressed positive views of CCM services. Practice respondents also perceived several patient benefits, including improved adherence to treatment, access to care team members, satisfaction, care continuity, and care coordination. Facilitators of CCM provision included having an inpractice care manager, patient-centered medical home recognition, experience developing care plans, patient trust in their provider, and supplemental insurance to cover CCM copayments. Most billing practices reported few problems obtaining patients' consent for CCM, though providers felt that CMS could better facilitate consent by marketing CCM's goals to beneficiaries. Barriers reported by professional society representatives and by billing and non-billing providers included inadequacy of CCM payments to cover upfront investments for staffing, workflow modification, and time needed to manage complex patients. Other barriers included inadequate infrastructure for health information exchange with other providers and limited electronic health record capabilities for documenting and updating care plans. Practices owned by hospital systems and large medical groups faced greater bureaucracy in implementing CCM than did smaller, independent practices.

CONCLUSIONS: Improving providers' experiences with and uptake of CCM will require addressing several challenges, including the upfront investment for CCM set-up

Electronic supplementary material The online version of this article (doi:10.1007/s11606-017-4134-7) contains supplementary material, which is available to authorized users.

Received December 15, 2016

Revised May 25, 2017

Accepted July 6, 2017

Published online July 28, 2017 and the time required to provide CCM to more complex patients.

KEY WORDS: care management; chronic care; coordination of care; primary health care; health care delivery system; payment policy. J Gen Intern Med 32(12): 1294-300 DOI: $10.1007 / \mathrm{s} 11606-017-4134-7$ (C) Society of General Internal Medicine 2017

\section{INTRODUCTION}

The rapidly evolving health policy climate has witnessed numerous efforts to improve the quality of care while reducing expenditures. ${ }^{1,2}$ Chronic care management (CCM), a common component of these efforts, is intended to enhance care continuity, care coordination, and ongoing management of patients' health conditions - much of which happens outside of the traditional office visit. Historically, Medicare has not compensated providers directly for care management performed outside of office visits, but providers argue that visit-based payments alone undervalue the work they do for patients between visits. ${ }^{3}$ Furthermore, a growing percentage of primary care providers experience burnout ${ }^{4}$ and lack adequate resources to manage patients' multiple chronic conditions.

In January 2015, the Centers for Medicare \& Medicaid Services (CMS) introduced the CCM payment policy (Current Procedural Terminology [CPT] code 99490) into the physician fee schedule. ${ }^{5}$ This new policy attempts to provide better financial support for primary care-based coordination and care management by reimbursing "eligible professionals" (e.g., physicians, nurse practitioners, and physician assistants, referred to as "providers" in this paper) for CCM services provided to patients with two or more chronic conditions. These conditions (such as congestive heart failure and diabetes) must place the patient at significant risk of death, acute exacerbation or decompensation, or functional decline, and must be expected to last at least 12 months. ${ }^{6-8}$

CCM scope-of-service requirements are similar to those of most patient-centered medical home (PCMH) initiatives. Although CCM participation does not require the timeconsuming PCMH recognition or certification process, ${ }^{9,10}$ practices must still ensure they have the infrastructure to deliver CCM. As with other Medicare payments, CCM is subject to audit. Although PCMH payment initiatives 
automatically reimburse practices on a per-member/per-month (PMPM) basis, under CCM, providers must bill Medicare for each beneficiary each month that they deliver CCM services. Furthermore, CCM payment is available only for patients who meet the eligibility criteria.

Under the CCM payment policy, providers may bill for 20 minutes of clinical staff time once per month per patient for non-face-to-face care management and coordination. Patients must provide a one-time written consent. The CCM reimbursement is approximately $\$ 42$. As with other Part B services, patients have a $20 \%$ coinsurance payment of approximately $\$ 8.00$, which is typically covered for patients with a Medigap policy or dual enrollment in Medicare and Medicaid. Before billing for CCM, the provider must have an initiating visit with the patient, which could be an annual wellness visit, an initial preventive examination, or a comprehensive evaluation and management visit.

To bill for CCM, the practice must assign a designated clinician to each patient, develop and maintain a comprehensive care plan in collaboration with the patient, communicate with other treating health professionals, and manage medications (Table 1). Practices must also have a certified electronic health record (EHR), make the care plan available electronically at all times to practice staff providing CCM, and share the care plan electronically outside the practice as appropriate. $^{6-8}$

During the first 15 months of the new payment policy, CCM uptake was low (4.5\%): 10,022 providers billed for CCM services, out of 224,187 non-institutional primary care providers that billed Medicare. ${ }^{11}$ These 10,022 providers worked in 3817 unique practices and billed for CCM for about 383,000 unique beneficiaries (approximately $2.4 \%$ of potentially eligible Medicare beneficiaries). ${ }^{12} \mathrm{CCM}$ beneficiaries received on average four months of CCM services (median of five months) in $2015 .^{12}$

Table 1 Chronic Care Management Scope of Services as Outlined in the January 2015 Policy

Structured Data Recording: patient demographics, problems, medications, allergies, and structured clinical summary in a certified EHR

Comprehensive Patient-Centered Care Plan: available to all practitioners within the practice who provide CCM, shared with patient and with other providers when appropriate

Access to Care: $24 / 7$ access to care management services; providing patient with a means to make timely contact with health care practitioners in the practice who have access to patient's EHR

Continuity of Care: designated practitioner or member of the care team with whom patient can get successive routine appointments

Enhanced Communication: opportunities for patient/caregiver with the practitioner (e.g., phone, secure messaging)

Management of Care: systematic assessment of patient's medical, functional and psychosocial needs; system based approaches to ensure timely preventive services; medication reconciliation; oversight of patient self-management of medications

Management of Care Transitions: among providers and settings including referrals to other providers, follow-up after: ED visit, hospital, skilled nursing facility or other facility discharge

Coordination of Care: with home and community based clinical service providers
Little is known about these providers' experience with CCM. To better understand their experiences, we describe the findings from semi-structured interviews with professional society representatives, billing and non-billing providers, and practice staff delivering CCM services. We examine respondents' perceptions of and experiences with CCM, facilitators of and barriers to CCM provision and billing, perceptions of its impact on patient outcomes, and the potential implications for CCM going forward. Such information can help CMS spend Medicare dollars wisely, help providers coordinate and manage patient care, and maximize the benefit patients receive from CCM.

\section{METHODS}

Interview topics. The interview topics (see online appendix) addressed the following: (1) How do practice characteristics, like size and ownership, affect practices' ability to provide CCM services? (2) How do practices provide and bill for $\mathrm{CCM}$, including changes in processes, resources, and personnel? (3) What are the barriers to and facilitators of providing and billing for CCM? (4) What are respondents' opinions on the adequacy of the CCM payment? (5) How do providers perceive beneficiaries' reactions to CCM? (6) What are providers' perceptions of the impact of CCM on care continuity, care coordination, and the use of health care services?

Provider sampling process. To identify CCM-billing providers, we combined data from Medicare claims from January 2015 through September 2015 with data from the National Plan and Provider Enumeration System and the Provider Enrollment, Chain, and Ownership System to capture provider contact information. We stratified the entire sample by region and specialty (primary care physician, other specialty physician, nurse practitioner, and physician assistant). Balancing across strata, we randomly identified about 500 CCM-billing providers. From these, we purposely identified 400 who varied in rural/urban location, provider type, number of unique beneficiaries for whom they billed CCM claims, and gender. We asked professional society representatives for references to non-billing providers, because cold-calling them would have been inefficient.

We also interviewed representatives from several professional organizations: American Medical Association, American College of Physicians, American Academy of Family Medicine, American Geriatric Society, American Osteopathic Association, American Association of Nurse Practitioners, American Association of Physician Assistants, and Medical Group Management Association. These interviews shed light on organization members' experiences regarding the CCM payment policy, including why some providers were not billing for CCM. 
Data collection. We mailed letters to 100 CCM-billing providers from our sample of 400 providers each month from January through April 2016. Three to five days after each mailing, we called each practice up to three times to schedule an interview. Practices were not compensated for participation.

We interviewed the provider or other staff (e.g., the care manager) most knowledgeable about the practice's CCM service delivery (Table 2). Two senior researchers with extensive experience interviewing providers conducted the 30- to 60minute interviews. We also interviewed non-billing providers from four practices about their perceived barriers to CCM participation and billing.

Coding and analysis. Two research analysts recorded and transcribed all interviews, with respondents' permission, and verified the transcript using the recording. The lead researcher read every transcript for context. The research team iteratively developed a codebook based on the research questions and a conceptual framework for care management. ${ }^{13,14}$ Each team member independently coded the first five interviews, and met with the other members to compare coding; the team then revised the codebook and its definitions to facilitate consistent coding across coders. The two analysts coded the remaining interviews and continued to meet weekly to verify their coding. The researchers conducted frequent debriefings and peer review to maximize coding reliability and reduce researcher bias. ${ }^{13,15,16}$ We iteratively reviewed key themes from the data to identify when data saturation had occurred. ${ }^{17}$ Data were analyzed as a single group, with an indicator for respondent type (professional society representative, CCM biller, or CCM non-biller) attached to each coded unit of text.

We used Atlas.ti ${ }^{18}$ to create data reports from the coded interviews. Each research team member read the query reports and outlined common themes, such as barriers to and facilitators of CCM implementation and workflow changes needed to implement CCM.

This study is exempt (45 CFR 46.101[b]) from institutional review board review because it was conducted subject to the approval of the head of CMS and was designed to evaluate the CCM services program.

\section{RESULTS}

\section{Practice Characteristics}

We completed 71 interviews: 56 with respondents from billing practices, 4 with providers from non-billing practices, and 11 with professional society representatives. Around the 55th practice interview, we reached data saturation, hearing no new information from respondents. ${ }^{17}$ We completed another five interviews ( 60 total) to ensure variation in practice characteristics. The four interviews with non-billing providers made it clear that perceived barriers to and facilitators of
Table 2 Characteristics of Respondents and Their Practices

\begin{tabular}{|c|c|}
\hline Respondent characteristics & Number \\
\hline Total completed interviews & 71 \\
\hline Professional society representatives & 11 \\
\hline Respondents from billing practices & 56 \\
\hline Respondents from non-billing practices & 4 \\
\hline \multicolumn{2}{|l|}{ Type of respondent (spread across a total of 50 practices)* } \\
\hline Physician (MD or DO) & 39 \\
\hline Nurse practitioner & 4 \\
\hline Physician assistant & 2 \\
\hline Nurse (RN LPN), social worker, or medical assistant & 7 \\
\hline Other (practice manager, business officer, etc.) & 8 \\
\hline \multicolumn{2}{|l|}{ Respondent gender } \\
\hline Female & 31 \\
\hline Male & 29 \\
\hline \multicolumn{2}{|l|}{ Practice characteristics ${ }^{\dagger *}$} \\
\hline \multicolumn{2}{|l|}{ Respondents' practice locations } \\
\hline Urban/suburban & 32 \\
\hline Rural & 18 \\
\hline \multicolumn{2}{|c|}{ Number of practices from the 6 states with highest CCM billing } \\
\hline Texas) & \\
\hline \multicolumn{2}{|l|}{ lexas) } \\
\hline Solo provider & 18 \\
\hline $2-5$ providers & 11 \\
\hline $6-10$ providers & 3 \\
\hline $11+$ providers & 18 \\
\hline \multicolumn{2}{|l|}{ Medical specialties of respondents' practices } \\
\hline Family practice medicine & 25 \\
\hline Internal medicine & 15 \\
\hline Geriatric medicine & 5 \\
\hline Cardiology & 5 \\
\hline \multicolumn{2}{|c|}{$\begin{array}{l}\text { Number of unique CCM beneficiaries in } 2015 \text { per billing provider } \\
\text { interviewed }\end{array}$} \\
\hline $1-5$ & 11 \\
\hline $6-25$ & 11 \\
\hline $26-100$ & 12 \\
\hline $101-499$ & 8 \\
\hline $500+$ & 1 \\
\hline Unknown" & 3 \\
\hline \multicolumn{2}{|l|}{ Ownership type } \\
\hline Physician-owned practice & 34 \\
\hline Hospital/system-owned practice & 16 \\
\hline \multicolumn{2}{|l|}{ Practice is recognized PCMH } \\
\hline Yes & 18 \\
\hline No & 32 \\
\hline
\end{tabular}

*We interviewed 60 respondents in 50 practices. In 13 of the 60 interviews, we interviewed the practice manager, nurse care manager or social worker, and/or another staff member who was heavily involved in their CCM program because the provider (physician, nurse practitioner, or physician's assistant) was unavailable for an interview

${ }^{*}$ Practice characteristics of the 50 unique practices from which we interviewed respondents. In cases where we interviewed multiple respondents in one practice (for example, the practice manager and the provider from the same practice), we counted the practice only once ${ }^{*}$ The four non-billing providers are included in the counts. All four were physicians, one each in internal medicine, family medicine, geriatrics, and cardiology. Two were in urban/suburban areas and two in rural areas. Two were women, two were solo practitioners, one was in a practice of 6-10 providers, and one was in a practice of $11+$ providers. Three of the four were in physician-owned practices. Only one of the four was in a practice previously recognized as a patient-centered medical home

$\S$ "Providers" refers to physicians, nurse practitioners, and physician assistants eligible to bill for the chronic care management (CCM) fee

"Three of the CCM-billing providers interviewed were identified by referrals, and thus we lack the data on the total number of CCM claims they had filed

CCM were similar to those mentioned by billing providers and by the professional society representatives.

The 60 practice respondents came from 50 unique practices that varied in size, urban versus rural location, geography, 
ownership type, and respondent type and specialty (Table 2). Twenty-nine of the 50 practices had five or fewer providers, three had 6 to 10 providers, and 18 had 11 or more providers. Thirty-four (68\%) were independent physician-owned practices, and the remaining 16 (32\%) were system-owned. Over one-third of CCM-billing practices were in rural areas. This distribution was consistent with the national sample of CCM billers from our claims analysis. ${ }^{12}$ Eighteen $(36 \%)$ of the 50 practices were recognized patient-centered medical homes (PCMHs).

Practice size and ownership characteristics seemed to affect the level of interest in and ability to provide and bill for CCM services. Practices owned by hospital systems and large medical groups faced greater bureaucracy within their parent organizations in setting up CCM billing and documentation than did smaller, independent practices. As a professional society representative remarked, "It's more of a challenge for [providers in large group practices and hospital systems] to implement [CCM]. ...they are less nimble, they have less control over their practice. They have to wait for approvals, etc."

Professional society representatives lacked systematic data on their members' participation in CCM, but said they received more calls about CCM from members in small, independent practices than those from large or system-owned practices.

\section{Frequency of CCM Billing}

The CCM-billing patterns of providers in our qualitative sample were similar to those of CCM billers nationally. ${ }^{12}$ Most providers did not bill the CCM fee every month for CCMenrolled beneficiaries. Over the first 12 months of the CCM program, CCM-enrolled beneficiaries received an average of four months of CCM services (median $=5$, range 1-12). ${ }^{12}$

\section{How Practices Deliver CCM Services}

Care management and staffing models for delivering CCM services fell into four categories.

Care manager-led model. In 21 of the 50 practices, care managers led the CCM. In this model, the care manager - typically a nurse, nurse practitioner, or physician assistant — handles most CCM activities, like enrollment, care plan creation, and communication with patients between visits. The care manager's interaction with the provider was usually limited to signing off on the initial care plan or addressing exacerbations of a patient's condition between visits.

Shared-responsibility model. In 15 practices, both providers and care managers played large roles in CCM. The provider led the CCM team but delegated tasks to care managers or other clinical staff. Care managers conducted many CCM activities but met frequently with the provider to discuss patients. As one provider stated, "Our entire practice is involved with CCM. It is the heart and soul of what our practice does."
Physician-led model. In six practices, the provider handled most of the CCM responsibilities. This was especially common among solo practitioners who understood their practice's clinical and administrative activities. Some handled CCM activities because of a combination of challenges, including the inability to afford a care manager, a shortage of qualified care managers, and little time to train other staff in care management. These practices often relied on medical assistants to handle less clinically complex aspects of care management.

Third-party model. Although many respondents described having been approached by third-party vendors to provide CCM services, only four practices used such vendors in 2015. These practices reported lacking the time to understand the CCM requirements or maintain the documentation required for CCM billing. Three of these four practices had stopped using the vendors because they felt the vendors contributed to fragmented care, created unnecessary paperwork, communicated poorly with the practice, and did not meaningfully improve the quality of care. Noting that patients disliked receiving calls from vendors, one provider stated, "If this was your mother, who would she respond better to? Some call center in Wichita, Kansas, or Suzie who has worked in my office for years and knows the patient and her frailties?" After dropping the vendors, the practices used existing staff or hired a care manager to continue CCM services.

\section{Barriers to Providing and Billing for CCM}

Barriers to providing CCM fell into four categories: changes in personnel and resources, inadequate EHR functionalities and interoperability, provider concerns about CCM requirements, and low-income patients' inability to afford the CCM copay.

Changes in personnel and resources were greatest for between-visit outreach to patients for chronic condition management, care transitions, and the creation and use of comprehensive care plans. Providers valued between-visit outreach and care transition monitoring, noting that doing this efficiently required hiring a care manager, and for larger practices, more than one care manager. A few practices repurposed the role of an existing nurse or medical assistant, sometimes extending their hours from part to full time. Providers had mixed reactions to the changes in personnel and resources required to create and update comprehensive care plans for CCM. Practices that were previously creating care plans noted that they required minor modifications. A few practices that had not previously created care plans were able to develop them. But some practices that had not previously created plans reported that the CCM plan components defined by CMS were "overly complex," "not clinically useful," "timeconsuming," and "expensive." A few providers felt burdened by care plans, because they believed that the rule did not allow billing for time spent creating the plans.

The lack of a unified infrastructure for health information exchange between providers posed a barrier to managing care 
transitions and sharing care plans, particularly for independent practices. A non-billing provider noted, "The idea that everyone must have access to the care plan is fine if everyone is using the same EHR, but many are not." Respondents also reported that hospitals hesitated to share patient information with practices due to concerns about violating the Health Insurance Portability and Accountability Act. However, respondents in large systems where providers used the same EHR reported better support for $24 / 7$ electronic access to care plans and care transitions.

Some respondents felt that the "copious documentation" needed for CCM was burdensome. Practice staff reported time-consuming workarounds and duplicate data entry between their EHR and care management software used for care plans, tracking CCM activities, and tracking time spent on non-faceto-face tasks. A family practitioner commented that, although CCM was worthwhile, "it takes 20 minutes to simply do the work around the work." Some practices purchased an EHR add-on module to support documentation of CCM tasks (such as time tracking and billing). Others used separate software such as Microsoft Access or Excel to track CCM information. Some practices relied on the care manager or practice manager to handle all CCM documentation in the EHR.

Provider perceptions and fears, particularly about mentioning the letter of consent and copay to patients, hindered CCM uptake. These barriers were most commonly reported by professional society representatives and non-billing providers, who feared that asking patients to provide consent and to cover the copay would damage the doctor-patient relationship. Although some billing providers had shared this concern before CCM implementation, most reported that their practices had found successful ways to discuss CCM with patients.

Most billing practices drafted their own consent letter, often after referring to sample letters published online by professional societies. A few practices mailed the letter to patients, but most approached the patient for consent during an office visit. Two billing practices reported not asking patients for consent because they felt uncomfortable doing so or were unaware of the requirement. Two other billing providers stated that they would discontinue care for eligible Medicare patients who did not consent to CCM, because they could not sustain the costs of providing care management and coordination without reimbursement.

Providers also reported that patients, particularly those with low incomes and no supplemental coverage, felt burdened by the copay. A billing physician noted, "The copayment is an issue for needy groups... [like those] who live on Social Security alone."

Practices that believed the CCM policy disallowed billing for a medical assistant's time felt a strain on their financial and staffing resources. In these practices, licensed practitioners performed simple tasks that medical assistants had previously done, such as routine patient education for less complex patients and phone outreach after emergency department (ED) visits.

\section{Facilitators of Providing and Billing for CCM}

Facilitators of CCM included prior experience as a recognized $\mathrm{PCMH}$ and with care plans, and support from professional societies.

Practices with experience with the CCM requirements, including those with PCMH recognition, faced the fewest challenges in billing for CCM. Echoing the comments of other billing providers, one internist said, "We spent a lot of time on the phone with our chronically ill patients. It was good news that we could finally be paid for it." Such practices had experience developing care plans, customizing the EHR to accommodate new workflows, providing care management services through a qualified care manager, and communicating with patients through a patient portal. Several practices used CCM modules in their EHR, which they said greatly facilitated CCM billing. Most billing practices lacked an existing care plan tool in their EHR and benefited from access to health information technology experts who could create customized care plan templates.

Providers commonly noted that professional societies supported their understanding of CCM requirements and facilitated CCM uptake. Support from professional societies included training and materials on CCM fee requirements, strategies for informing patients about the copay, and templates for the consent letter and care plans.

\section{Adequacy of the CCM Payment}

Respondents welcomed the CCM payment, but many felt that the reimbursement did not cover the necessary investments. For example, setting up the infrastructure (e.g., workflows, EHR modifications) and staffing for CCM required a high initial investment that small practices especially could not afford. Geriatricians and some general internists in particular felt that the CCM payment did not cover the cost of developing care plans and managing complex patients. Some respondents reported that practice staff usually spent 45 to 60 minutes on each CCM patient each month-and several hours on the most complex patients. Two independent practices, however, had enrolled most of their eligible patients in CCM and credited the CCM payment for improving care and making it possible to accept Medicare patients while staying independent.

\section{Beneficiaries' Reactions to CCM}

Practice respondents noted that CCM patients had overwhelmingly positive views of these services. Patients appreciated having a dedicated point of contact and increased telephonic and electronic access to their providers.

The few patients who reacted negatively to CCM typically lacked supplemental insurance to cover the copay or assumed that practices would provide this care regardless of compensation. A provider noted, "Families and patients think that [CCM] is a cash cow. Medicare never told them the value of this code and why providers should bill for it." Several providers suggested that CMS should promote CCM to patients to 
better educate them about the service and reduce the burden on providers to "sell the program."

\section{Impact of CCM on Continuity and Coordination of Care}

Most respondents reported that CCM payments improved care coordination at their practice by enabling more outreach to patients, specialists, and hospitals. One family practitioner said, "[CCM] is another way to put our eyes on patients; we monitor them closely." Although some providers had initial concerns that CCM might hinder patient-primary care provider continuity (if other specialists billed for CCM services), only one provider had experienced this problem.

\section{Perceived Impact of CCM on Outcomes}

Providers reported that CCM resulted in several positive patient outcomes, including improved satisfaction, access to the practice, adherence to treatment recommendations, and enhanced time management, allowing the provider to focus on the patient during office visits. An internist said, "We have done [CCM] in our practice and surveyed our patients. The patient sees it as a very desirable service for the practice to have." Some providers noted that CCM funding enabled them to hire a nurse to do pre-visit planning, which allowed the provider to focus on the patient during the visit rather than wasting time retrieving lab results and other data. They also noted that monitoring patients before and after visits allowed them to address patients' issues as they occurred; one care manager said, "We have quite a few patients who require extra help managing their [chronic conditions].... What I found is that talking with the patient, or giving the patient the opportunity to call us so they can talk to us about their concerns, helps us keep them out of the emergency room." Respondents also reported that care managers connected patients with community-based services that could improve their health and quality of life. As one provider said, "I think it's a great program. It decreases hospitalizations, increases compliance. Overall [it] helps everyone."

\section{DISCUSSION}

In sum, CCM-billing providers and practice staff perceive $\mathrm{CCM}$ as having several benefits for patients. It encourages adherence to treatment, provides better access to care team members, and improves the continuity and coordination of care, as well as satisfaction. Several factors make CCM easier to provide, such as a care manager at the practice who is dedicated solely to CCM, prior recognition as a medical home, experience developing care plans, and patients who trust their provider and who have supplemental insurance to cover copayments. The barriers to $\mathrm{CCM}$ range from inadequate payment for CCM services to problems obtaining patients' consent, inadequate infrastructure for data exchange between CCM-billing practices and other providers, and EHRs that cannot support the development and maintenance of care plans.

Unlike PCMH initiatives, CCM does not send practices automatic PMPM payments; it requires additional billing and time-tracking work for practices. CCM also requires greater effort in enrolling patients and obtaining consent. Moreover, patients receiving $\mathrm{CCM}$ are responsible for the Part $\mathrm{B}$ coinsurance. Although this is covered by most Medigap policies, for those without supplemental coverage, the coinsurance created a barrier to CCM.

Several billing and non-billing providers noted that CCM payments do not cover the large upfront investments required to hire a care manager, change workflows, and manage complex patients. According to a microsimulation model, a practice would need to enroll 131 Medicare patients in CCM and bill for their monthly CCM services under the 2015 payment amount to recoup the costs of hiring a full-time registered nurse dedicated to providing CCM. The practice would need 76 Medicare patients to support a licensed practical nurse. ${ }^{19}$ However, most practices we interviewed enrolled far fewer patients in CCM and had only billed for a few months per patient.

A recent article ${ }^{20}$ summarized conversations with several physicians about CCM and noted that approaching patients about CCM and obtaining consent is most successful if done by the patient's regular physician rather than another member of the practice. This was the case in our study as well. Twiddy et al. also provide useful examples of how some practices are handling care plans and time tracking for $\mathrm{CCM}^{20}$

To support the adoption of CCM, CMS recently published its changes to the CCM payment policy, which took effect January 1, $2017,{ }^{21}$ and may help address several barriers identified in our study. First, to address providers' concerns about payment for the delivery of CCM to complex patients, CMS introduced new billing codes to increase the payment amounts for CCM. Besides the existing CCM CPT code 99490, which is still used for patients who have two or more chronic conditions but not complex medical needs, CMS created an add-on code (G0506, which pays approximately \$64) for the CCM initiating visit. This code is for any assessments and CCM care planning beyond the usual effort involved in the separately billable CCM initiating visit. CMS also has two new CPT codes for complex patients (CPT 99487, which pays $\$ 94$ for 60 minutes of clinical staff time, and CPT 99489, which pays $\$ 47$ for each additional 30 minutes of clinical staff time). Second, CMS will allow oral rather than written patient consent as long as it is documented in the patient's record. Third, CMS has reduced the technology requirements to allow providers to focus on the timely exchange of health information - the care plan and transitional care documents - between the CCM-billing practice and outside providers, rather than requiring CCM providers to provide $24 / 7$ electronic access for these pieces. Practices must still use a certified EHR for the structured recording of key patient information.

Another concern raised prior to CCM implementation was the possibility that practices would stop caring for patients 
who refused to consent to $\mathrm{CCM}^{9}{ }^{9}$ Of the 50 practices we interviewed, two indicated that they would do this. Although rare, this could be monitored going forward to identify whether this is an unintended consequence.

Even with CMS's proposed revisions, providers will likely continue to struggle to establish and maintain comprehensive care plans as envisioned in CCM policy. In our study, as in prior work, ${ }^{22}$ providers and care managers note that current EHRs, including some with add-on care management software, did not help them create or update useful, clinically meaningful care plans.

A limitation of this study is that we interviewed only four non-billing providers, although the themes they raised were also raised by professional society representatives and billing providers. The study's main strength is the nuanced information obtained from an array of practices and professional societies. Although policy-oriented organizations typically respond to opportunities for public comment to CMS, our findings provide a systematic analysis of the perspectives of realworld providers to the debate.

Looking ahead, policymakers and private insurers should explore ways to encourage uptake of and reimbursement for primary care-based CCM, given that prior work demonstrates that chronic care management improves patient outcomes and reduces ED use and hospitalizations. ${ }^{23,24}$ Further studies should also explore changes in patients' experience, care utilization, and health as a result of improved CCM. Finally, as non-face-to-face $\mathrm{CCM}$ increases in prevalence, researchers can examine whether this practice is narrowing the gap in care in rural areas where patients must travel long distances for office visits or where there are shortages of health professionals.

Acknowledgements: Data collection and analysis were performed under a contract with the Centers for Medicare \& Medicaid Services.

Corresponding Author: Ann S. O'Malley, MD, MPH; Mathematica Policy Research, 1100 First St., NE, 12th Floor, Washington, DC 20002-4221, USA (e-mail: aomalley@mathematica-mpr.com).

\section{Compliance with Ethical Standards:}

Funding: Funding for data collection and analysis was provided under a contract with the Centers for Medicare \& Medicaid Services.

Conflict of Interest: The authors declare that they do not have a conflict of interest.

\section{REFERENCES}

1. Centers for Medicare and Medicaid Services. Innovation Models. Available at: https://innovation.cms.gov/initiatives/\#views=models. Accessed July 1 , 2017.

2. Meyers D, Peikes D, Genevro J, et al. The Roles of Patient-Centered Medical Homes and Accountable Care Organizations in Coordinating Patient Care. AHRQ Publication No. 11-M005-EF. Rockville, MD: Agency for Healthcare Research and Quality. December 2010. Available at: https: / / pcmh.ahrq.gov/sites/default/files / attachments / Roles\%20of\%20PCMHs\%20And\%20ACOs\%20in\%20Coordinating\%2OPatient\%20Care.pdf. Accessed July 1, 2017.
3. Press MJ. Instant replay—a quarterback's view of care coordination. N Engl J Med. 2014;371:489-91.

4. Shanafelt TD, Boone S, Tan L, et al. Burnout and satisfaction with work-life balance among U.S. physicians relative to the general U.S. population. Arch Intern Med. 2012;172(18):1377-85.

5. Current Procedural Terminology code 99490

6. CMS-1612-FC. "CY 2015 Revisions to Payment Policies Under the Physician Fee Schedule and Other Revisions to Medicare Part B." Nov. 13, 2014. Available at http://www.cms.gov/ Mdicare/MedicareFee-for-Service-Payment/PhysicianFeeSched/PFS-Federal-RegulationNotices-Items/CMS-1612-FC.html. Accessed July 1, 2017

7. CMS-81 FR 46161. Federal Register. Available at: http://www. federalregister.gov/articles/2016/07/15/2016-16097/medicare-program-revisions-to-payment-policies-under-the-physician-fee-scheduleand-other-revisions\#h-109. Accessed July 1, 2017.

8. Department of Health and Human Services, Centers for Medicare and Medicaid Services. Medicare Learning Network. "Chronic Care Management Services.” ICN 909188 January 2015. Available at: https://www.cms.gov/ Outreach-and-Education/Medicare-Learning-Network-MLN/MLNProducts/ Downloads/ChronicCareManagement.pdf. Accessed July 1, 2017.

9. Edwards ST, Landon BE. Medicare's chronic care management payment-payment reform for primary care. N Engl J Med. 2014;371(22):2049-51.

10. Halladay JR, Mottus K, Reiter K, et al. The Cost to Successfully Apply for Level 3 Medical Home Recognition. J Am Board Fam Med. 2016;29(1):69-77. doi:10.3122/jabfm.2016.01.150211.

11. CMS Fast Facts March 2017 Version. Available at: https://www.cms.gov/ Research-Statistics-Data-and-Systems/Statistics-Trends-and-Reports/ CMS-Fast-Facts/index.html. Accessed July 1, 2017.

12. Schurrer J, O'Malley AS, Wilson C, McCall N, Jain N. Evaluation of the Diffusion and Impact of the Chronic Care Management (CCM) Services: Interim Report. Washington, DC: Mathematica Policy Research; 2016.

13. Miles MB, Huberman AM, Saldana J. Qualitative data analysis: a methods sourcebook. 3rd ed. Thousand Oaks (CA): Sage Publications; 2013. 381 p.

14. Bodenheimer T, Berry-Millet R. Care management of patients with complex health care needs [Internet]. Princeton (NJ): Robert Wood Johnson Foundation; 2009 Dec [cited 2016 Nov 9]. 40 p. Research Synthesis Report No.19. Available at: http://www.rwjf.org/en/library/ research/2009/12/care-management-of-patients-with-complex-healthcare-needs.html. Accessed July 1, 2017.

15. Cohen DJ, Crabtree BF. Evaluative criteria for qualitative research in health care: controversies and recommendations. Ann Fam Med. 2008;6(4):331-39.

16. Lincoln YS, Guba EG. Naturalistic inquiry. Newbury Park: Sage Publications; 1985.

17. Guest G, Bunce A, Johnson L. How many interviews are enough? An experiment with data saturation and variability. Field Methods. 2006;18(1):59-82.

18. ATLAS.ti Qualitative Data Analysis software, version 7.5.10.

19. Basu S, Phillips RS, Bitton A, Song Z, Landon BE. Medicare chronic care management payments and financial returns to primary care practices: a modeling study. Ann Intern Med. 2015;163(8):580-88.

20. Twiddy D. Chronic Care Management in the Real World. Fam Pract Manag. 2015;22(5):35-41.

21. Department of Health and Human Services, Centers for Medicare and Medicaid Services. 80170 Federal Register / Vol. 81, No. 220 / Tuesday, November 15, 2016 / Rules and Regulations. Available at: https://www.gpo. gov/fdsys/pkg/FR-2016-11-15/pdf/2016-26668.pdf. Accessed July 1, 2017.

22. O'Malley AS, Draper $\mathbf{K}$, Gourevitch R, Cross DA, Scholle SH. Electronic health records and support for primary care teamwork. J Am Med Inform Assoc. 2015;22(2):426-34.

23. Urato C, McCall N, Cromwell J, Lenfestey N, Smith K, Raeder D (RTI International; Research Triangle Park, NC). Evaluation of the extended Medicare Care Management for High Cost Beneficiaries (CMHCB) Demonstration: Massachusetts General Hospital (MGH): final report [Internet]. Baltimore (MD): Centers for Medicare and Medicaid Services; 2013 Oct. (RTI Project No. 0209853.018). Available at: http://www. massgeneral.org/News/assets/pdf/FullFTIreport.pdf. Accessed July 1, 2017.

24. Hsu J, Price M, Vogeli C, Brand R, Chernew ME, Chaguturu SK, Weil E, Ferris TG. Bending The Spending Curve By Altering Care Delivery Patterns: The Role Of Care Management Within A Pioneer ACO. Health Aff (Millwood). 2017;36(5):876-884. 